\section{Uso de oxigenador de membrana extracorpórea venoarterial en un paciente con fallo del ventrículo derecho operado \\ de tromboendarterectomía pulmonar}

\section{Veno-arterial extracorporeal membrane oxygenation support in a patient with right ventricular failure post- pulmonary endarterectomy surgery}

La hipertensión pulmonar tromboembólica crónica (HPTEC) se clasifica en el grupo iv del reciente Consenso de hipertensión pulmonar realizado en Niza, Francia ${ }^{1}$. Se caracteriza por trombos crónicos organizados adheridos a la capa media de la arteria pulmonar que obstruyen su luz ${ }^{2}$.

Para su diagnóstico se requiere obtener por cateterismo cardiaco derecho la presión en la arteria pulmonar (PAP) media $\geq 25 \mathrm{~mm} \mathrm{Hg}$, presión capilar pulmonar $\leq 15 \mathrm{~mm} \mathrm{Hg}$ y la evidencia de al menos uno o más defectos de perfusión por métodos de imagen $^{1,2}$.

La tromboendarterectomía pulmonar (TEnd) es el procedimiento quirúrgico de elección para resolver la HPTEC $^{1,2}$, y requiere del uso de circulación extracorpórea, hipotermia y paro circulatorio ${ }^{3}$. La supervivencia a los 5 años es del $82 \%$ y a los 10 años del $75 \%^{4}$. La mortalidad postoperatoria se acepta que sea $<5 \%^{1}$ para ser considerada centro de la referencia.

Las complicaciones asociadas a morbimortalidad son debidas a lesión por reperfusión, hemorragia pulmonar masiva, fallo del ventrículo derecho (VD) e hipertensión pulmonar persistente o residual ${ }^{3,5-9}$. En consecuencia, el retiro de la derivación cardiopulmonar (DCP) se torna difícil e incluso imposible, ya que lleva al fallecimiento del paciente. En estas condiciones el uso de oxigenador de membrana extracorpórea (ECMO, por sus siglas en inglés) puede utilizarse como un recurso más para el tratamiento de estos pacientes graves, ya que les proporciona soporte vital avanzado de tipo cardiopulmonar y tiempo para la recuperación del órgano ${ }^{6}$.

Actualmente hay reportes en la literatura que han sugerido el uso de ECMO venoarterial (ECMO-VA) como puente a la recuperación en aquellos pacientes que presentan estos tipos de complicaciones en el transoperatorio o posoperatorio inmediato ${ }^{5-9}$.

Presentamos en esta carta científica el primer caso exitoso informado en nuestro país con el uso de ECMO-VA para el tratamiento del fallo del VD posterior a TEnd. En la literatura internacional se ha informado su utilidad en el fallo del VD post-TEnd solo en 2 trabajos previos ${ }^{8,9}$.

Un hombre de 33 años de edad con antecedentes de sedentarismo, obesidad y tabaquismo, con cuadro clínico de 2 meses de evolución caracterizado por episodio de disnea súbita y síncope, fue referido a nuestra unidad por sospecha de tromboembolia pulmonar.

$\mathrm{Al}$ ingreso en urgencias se documentó saturación arterial de oxígeno $\left(\mathrm{SaO}_{2}\right)$ del $85 \%$ al aire ambiente, presión arterial sistémica (PAS) $(120 / 80 \mathrm{~mm} \mathrm{Hg})$ y el electrocardiograma con frecuencia cardiaca de 72 lat/min, desviación del eje a la derecha, bloqueo incompleto de rama derecha del haz de His, imagen $S_{1} Q_{3} T_{3}$ y sobrecarga sistólica del ventrículo derecho.

En un ecocardiograma transtorácico se le encontró signo de McConell, dilatación de cavidades derechas, tronco y ambas ramas de la arteria pulmonar, la presión sistólica de la arteria pulmonar estimada en $61 \mathrm{~mm} \mathrm{Hg}$ y excursión sistólica del plano del anillo tricuspídeo (TAPSE, por sus siglas en inglés) $16 \mathrm{~mm}$, índice de excentricidad del VD 1.9.

La angiotomografía de ingreso mostró datos en relación con trombosis de la rama arterial pulmonar izquierda y de ramos segmentarios superior e inferior de la arteria pulmonar derecha, derrame pleural derecho, nódulo pulmonar subpleural izquierdo en segmento posterobasal. Se decidió fibrinólisis con tenecteplase $50 \mathrm{mg}$ iv en dosis única. Se tuvo mejoría parcial del cuadro clínico y en la angiotomografía de control (fig. 1A) permaneció la imagen de oclusión vascular pulmonar bilateral. Por este motivo se consideró candidato a TEnd en sesión médico-quirúrgica.

En el transoperatorio se apreció cardiomegalia grado ॥ a expensas de cavidades derechas con dilatación del tronco y ambas ramas de la arteria pulmonar. Además se encontró un gran trombo organizado con extensión hacia las 2 ramas de la arteria pulmonar que en las arterias lobares izquierdas las obstruía en su totalidad. La red venosa colateral se estimó abundante.

El tiempo de derivación cardiopulmonar fue de $134 \mathrm{~min}$, pinzamiento aórtico de $76 \mathrm{~min}$, paro circulatorio con hipotermia profunda a $18{ }^{\circ} \mathrm{C}$ de $25 \mathrm{~min}$ y sangrado total de $625 \mathrm{ml}$.

Posterior al retiro de la derivación cardiopulmonar presentó disminución de la $\mathrm{SaO}_{2}$ al $40 \%$, aumento de la PAP a $88 \mathrm{~mm} \mathrm{Hg}$ y dilatación del VD con disminución de la PAS a $46 \mathrm{~mm} \mathrm{Hg}$, por lo que se decidió en ese momento la colocación de ECMO-VA (fig. 1B) (sistema ECMO QUADROX PLS, Rotaflow -Maquet Cardiovascular, Hirrlingen, Alemania-), mediante disección de vena y arteria femorales. Se accedió a la vena mediante técnica de Seldinger y con anastomosis término-lateral e injerto de dacrón $8 \mathrm{~mm}$ para la arteria, la cual se fijó directamente a una cánula aórtica arterial 22 Fr y a la línea arterial del dispositivo (fig. 1C), con lo que mejoró su PAS media a $89 \mathrm{~mm} \mathrm{Hg}$, la $\mathrm{SaO}_{2}$ al $92 \%$ y la PAP media a $35 \mathrm{~mm} \mathrm{Hg}$ posterior a la colocación del ECMO-VA.

En la terapia intensiva el comportamiento fue hacia la mejoría en sus parámetros de laboratorio, hemodinámicos y del intercambio gaseoso, como se muestra en la tabla 1.

El tratamiento con vasodilatadores pulmonares se realizó con óxido nítrico inhalado a razón de 20 ppm, sildenafil $20 \mathrm{mg}$ vo cada $8 \mathrm{~h}$ y milrinona iv a dosis variables.

Debido a evolución favorable se decidió el retiro del ECMO-VA $48 \mathrm{~h}$ después de la cirugía. Así también se inició el retiro de la milrinona iv, del óxido nítrico y se sustituyó con iloprost inhalado a razón de $5 \mathrm{mcg}$ cada $4 \mathrm{~h}$.

Presentó neumonía asociada a la ventilación, la cual fue tratada con esquema a base de imipenem/amikacina. Al sexto día postoperatorio se extubó; fue egresado de la terapia intensiva y permaneció en hospitalización 15 días más. 


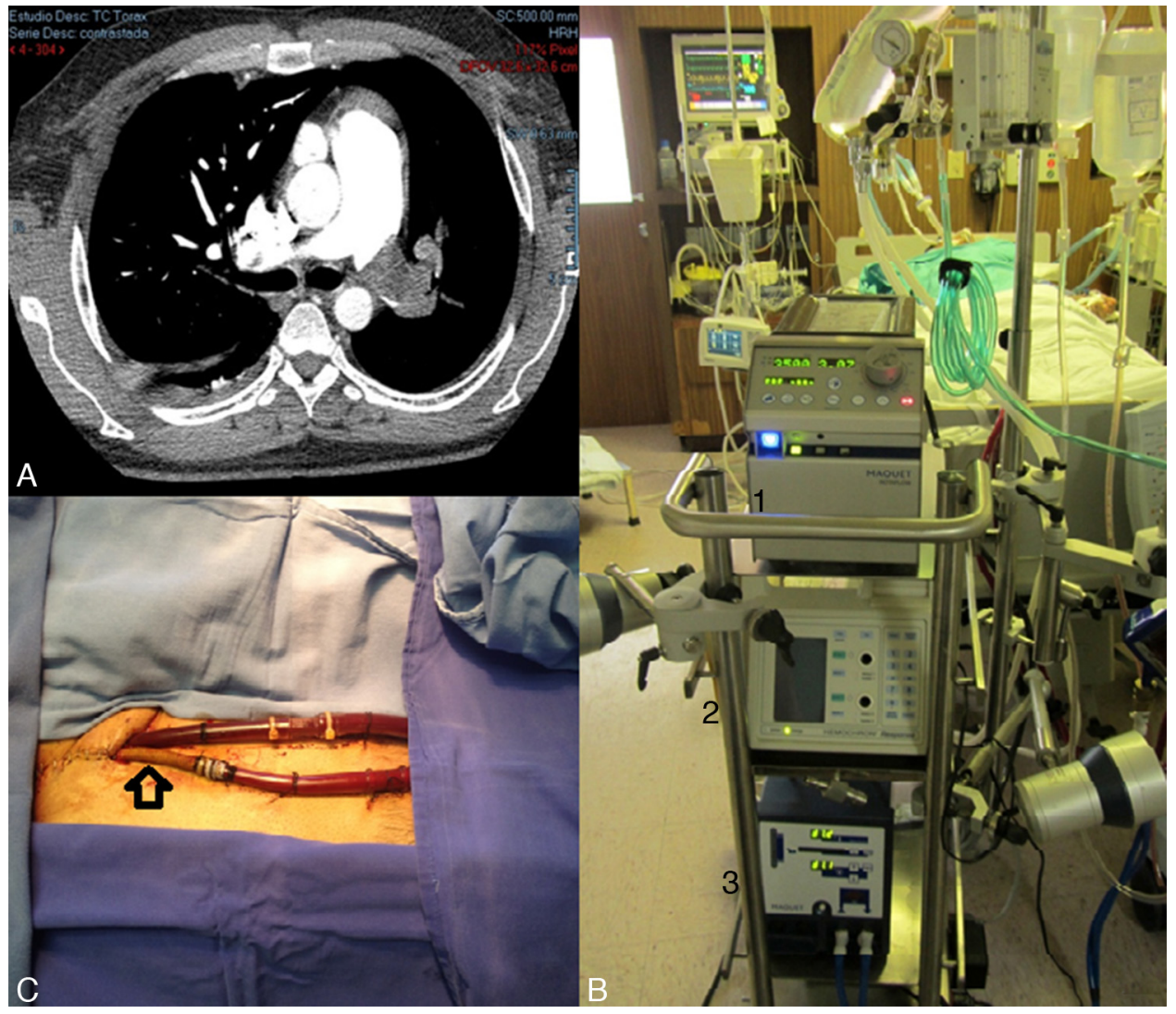

Figura 1 A. Tomografía axial computarizada donde se aprecia obstrucción de ambas ramas de la arteria pulmonar posfibrinólisis. B. Componentes del oxigenador de membrana extracorpórea: 1. consola del oxigenador de membrana extracorpórea; 2 . monitor de tiempo de coagulación; 3. intercambiador de temperatura. C. Cánulas arteriales con injerto de dacrón (flecha negra) y venosa.

La TEnd constituye el tratamiento de elección para la HPTEC de arterias proximales ${ }^{1-3}$. Los resultados obtenidos en este grupo de pacientes se consideran excelentes debido a mejoría en la técnica quirúrgica y cuidados postoperatorios. Debido a la supervivencia actual se considera un procedimiento curativo.

La mortalidad está relacionada con complicaciones perioperatorias como el sangrado pulmonar, la lesión pulmonar por reperfusión, la hipertensión pulmonar residual y el fallo del $V^{1,5-9}$. Su presencia puede hacer imposible el retiro de la derivación cardiopulmonar o complicar y prolongar el manejo de la ventilación mecánica.

En estas condiciones, en las que el tratamiento convencional no permite la supervivencia del paciente, el uso de ECMO es una alternativa que cada día es más aceptada ${ }^{1}$. La supervivencia al egreso hospitalario de estos pacientes, en los limitados estudios publicados con estas complicaciones, ha sido informado hasta del $57 \%$.

El paciente aquí presentado requirió apoyo mediante ECMO-VA secundario a fallo del VD posterior al retiro de la circulación extracorpórea. Su uso permitió la retirada de la derivación cardiopulmonar y estabilizar al paciente, como puede observarse en el comportamiento de las variables acotadas en la tabla 1.

El ECMO es una adaptación de la derivación cardiopulmonar en cirugía cardiaca y funciona como un corazón y pulmón artificial, ya que proporciona flujo sanguíneo a través de un circuito y una interface que sirve para el intercambio de dióxido de carbono y oxígeno ${ }^{10}$. El ECMO se ha categorizado por su uso como cardiaco, respiratorio o cardiorrespiratorio. De acuerdo al circuito puede ser venoarterial si el soporte es circulatorio y de intercambio gaseoso o venovenoso si solo es soporte del intercambio gaseoso el utilizado. En función del sitio de colocación puede ser central, conectado directamente al corazón o periférico a través de los vasos femorales $^{1}$ (fig. 1 C).

El utilizar un circuito venoarterial con respecto a uno venovenoso confiere las siguientes ventajas: 1) el circuito venoarterial permite «derivar» la circulación pulmonar, lo que reduce la presión pulmonar y evita el flujo sanguíneo a dicha zona; 2) mantiene una adecuada perfusión y oxigenación a los tejidos corporales, gracias al soporte cardiopulmonar que el dispositivo proporciona; 3) disminuye la sobrecarga al VD y la repercusión sobre la circulación sistémica por el fallo del VD; 4) debido al uso del oxigenador de membrana es posible también tratar la lesión por reperfusión pulmonar y la relacionada con la ventilación mecánica.

A manera de comentario final, en el fallo del VD visto en el postoperatorio de TEnd pulmonar el ECMO-VA puede ser utilizado para la retirada de la derivación cardiopulmonar. 
Tabla 1 Comportamiento de los valores de laboratorio, hemodinámicos y de la gasometría arterial posteriores a la colocación del oxigenador de membrana extracorpórea

\begin{tabular}{|c|c|c|c|}
\hline Variable & Día 1 & Día 2 & Día 6 \\
\hline $\begin{array}{l}\text { Hemoglobina, } \\
\text { g/dl }\end{array}$ & 12.6 & 11.3 & 10.2 \\
\hline Hematocrito, \% & 36.1 & 34 & 32 \\
\hline Plaquetas, K/ul & 246 & 141 & 365 \\
\hline $\begin{array}{l}\text { Leucocitos, } \\
\text { K/ul }\end{array}$ & 9.9 & 13.6 & 8.7 \\
\hline INR & 1.44 & 1.13 & 3.61 \\
\hline Glucosa, mg/dl & 183 & 135 & 78 \\
\hline Urea, mg/dl & 31.6 & 64.3 & 29 \\
\hline $\begin{array}{l}\text { Creatinina, } \\
\mathrm{mg} / \mathrm{dl}\end{array}$ & 1.4 & 1.5 & 0.7 \\
\hline $\begin{array}{l}\text { Presión venosa } \\
\text { central, } \\
\text { mm Hg }\end{array}$ & 24 & 15 & 12 \\
\hline $\begin{array}{l}\text { Presión arterial } \\
\text { pulmonar } \\
\text { media, } \\
\text { mm Hg }\end{array}$ & 30 & 22 & 15 \\
\hline $\mathrm{pH}$ & 7.33 & 7.40 & 7.41 \\
\hline $\mathrm{PaCO}_{2}, \mathrm{~mm} \mathrm{Hg}$ & 32 & 42 & 39 \\
\hline $\begin{array}{c}\mathrm{PaO}_{2} / \mathrm{FiO}_{2} \\
\mathrm{~mm} \mathrm{Hg} / \%\end{array}$ & 71 & 180 & 210 \\
\hline Lactato & 5.1 & 0.5 & 0.8 \\
\hline $\begin{array}{c}\text { Déficit de base, } \\
\mathrm{mmol} / \mathrm{l}\end{array}$ & 4.2 & 1.1 & 0.1 \\
\hline
\end{tabular}

\section{Financiación}

No se recibió patrocinio de ningún tipo para llevar a cabo este artículo.

\section{Conflicto de intereses}

Los autores declaran no tener ningún conflicto de intereses.

\section{Bibliografía}

1. Kim NH, Delcroix M, Jenkins DP, Channick R, Dartevelle P, Jansa $\mathrm{P}$, et al. Chronic thromboembolic pulmonary hypertension. J Am Coll Cardiol. 2013;62:D92-9.

2. Ghofrani HA, Distler O, Gerhardt F, Gorenflo M, Grünig E, Haefeli WE, et al. Treatment of pulmonary arterial hypertension (PAH): Updated recommendations of the Cologne Consensus Conference 2011. Int J Cardiol. 2011;154S:S20-33.
3. Thistlethwaite PA, Kemp A, Du L, Madani MM, Jamieson SW. Outcomes of pulmonary endarterectomy for treatment of extreme thromboembolic pulmonary hypertension. J Thorac Cardiovasc Surg. 2006;131:307-13.

4. Madani M, Auger W, Pretorius V, Sakakibara N, Kerr K, Kim N, et al. Pulmonary endarterectomy: Recent changes in a single institution's experience of more than 2,700 patients. Ann Thorac Surg. 2012;94:97-103.

5. Thistlethwaite A, Madani M, Kemp A, Hartley M, Auger W, Jamieson S, et al. Venovenous extracorporeal life support after pulmonary endarterectomy: Indications, techniques, and outcomes. Ann Thorac Surg. 2006;82: 2139-46.

6. Pretorius V, Alayadhi W, Modry D. Extracorporeal life support for the control of life-threatening pulmonary hemorrhage. Ann Thorac Surg. 2009;88:649-50.

7. Hou X, Xing J, Hao X, Li H, Gan H. Venoarterial extracorporeal membrane oxygenation support for two patients after pulmonary thromboendarterectomy. J Can Anesth. 2012;59: 622-3.

8. Berman M, Tsui S, Vuylsteke A, Snell A, Colah S, Latimer R, et al. Successful extracorporeal membrane oxygenation support after pulmonary thromboendarterectomy. Ann Thorac Surg. 2008;86:1261-7.

9. Ogino $\mathrm{H}$, Ando M, Matsuda H, Minatoya K, Sasaki H, Nakanishi $\mathrm{N}$, et al. Japanese single-center experience of surgery for chronic thromboembolic pulmonary hypertension. Ann Thorac Surg. 2006;82:630-6.

10. Hung M, Vuylsteke A, Valchanov K. Extracorporeal membrane oxygenation: Coming to an ICU near you. JICS. 2012;13: $31-8$.

Luis Raúl Meza-López ${ }^{\mathrm{a}}$, Luis Efren Santos-Martínez ${ }^{\mathrm{b}, *}$, Víctor Manuel Lozano-Torres ${ }^{a}$, Rutilio Daniel Jiménez-Espinosa ${ }^{a}$, Jesus Zaín Campos-Larios ${ }^{\mathrm{C}}$, Nielzer Armando Rodríguez-Almendros ${ }^{\mathrm{b}}$ y Moisés Cuttiel Calderón-Abbo ${ }^{d}$

a Departamento de Cirugía Cardiotorácica, UMAE Hospital de Cardiología del Centro Médico Nacional Siglo XXI, IMSS, México DF, México

${ }^{\mathrm{b}}$ Departamento de Hipertensión Pulmonar y Función Ventricular Derecha, UMAE Hospital de Cardiología del Centro Médico Nacional Siglo XXI, IMSS, México DF, México c Departamento de Terapia Intensiva Posquirúrgica Cardiovascular, UMAE Hospital de Cardiología del Centro Médico Nacional Siglo XXI, IMSS, México DF, México d Dirección General, UMAE Hospital de Cardiología del Centro Médico Nacional Siglo XXI, IMSS, México DF, México

* Autor para correspondencia: Avenida Cuauhtémoc n. ${ }^{\circ} 330$ Colonia Doctores, Delegación Cuauhtémoc, CP 06720, Distrito Federal, México.

Correo electrónico: luis.santosma@imss.gob.mx (L.E. Santos-Martínez).

http://dx.doi.org/10.1016/j.acmx.2014.11.001 